\title{
Cardiac arrest in spontaneous subarachnoid hemorrhage and associated outcomes
}

\author{
Eric Feldstein, MD, ${ }^{1}$ Jose F. Dominguez, MD, ${ }^{1}$ Gurkamal Kaur, BS, ${ }^{1}$ Smit D. Patel, MD, MPH, ${ }^{2}$ \\ Alis J. Dicpinigaitis, BA, ${ }^{1}$ Rosa Semaan, BS, ${ }^{1}$ Leanne E. Fuentes, BSN, RN, ${ }^{1}$ \\ Jonathan Ogulnick, BA, MusB, ${ }^{1}$ Christina Ng, BS, ${ }^{1}$ Cameron Rawanduzy, MS, ${ }^{1}$ Haris Kamal, MD, ${ }^{1}$ \\ Jared Pisapia, MD, ${ }^{1}$ Simon Hanft, MD, ${ }^{1}$ Krishna Amuluru, MD, ${ }^{3}$ Srihari S. Naidu, MD, ${ }^{4}$ \\ Howard A. Cooper, MD, ${ }^{5}$ Kartik Prabhakaran, MD, ${ }^{6}$ Stephan A. Mayer, MD, ${ }^{1}$ Chirag D. Gandhi, MD, ${ }^{1}$ \\ and Fawaz Al-Mufti, MD'
}

\begin{abstract}
${ }^{1}$ Department of Neurosurgery, Westchester Medical Center, New York Medical College School of Medicine; ${ }^{4}$ Department of Medicine, Westchester Medical Center, New York Medical College of Medicine; ${ }^{5}$ Department of Cardiology, Westchester Medical Center, New York Medical College of Medicine; ${ }^{6}$ Department of Surgery, Westchester Medical Center, New York Medical College of Medicine, Valhalla, New York; '2Department of Neurosurgery, University of California, Los Angeles, David Geffen School of Medicine, Los Angeles, California; and '3Department of Neurointerventional Radiology, Goodman Campbell Brain and Spine, Indianapolis, Indiana
\end{abstract}

OBJECTIVE The authors sought to analyze a large, publicly available, nationwide hospital database to further elucidate the impact of cardiopulmonary arrest (CA) in association with subarachnoid hemorrhage (SAH) on short-term outcomes of mortality and discharge disposition.

METHODS This retrospective cohort study was conducted by analyzing de-identified data from the National (Nationwide) Inpatient Sample (NIS). The publicly available NIS database represents a 20\% stratified sample of all discharges and is powered to estimate $95 \%$ of all inpatient care delivered across hospitals in the US. A total of 170,869 patients were identified as having been hospitalized due to nontraumatic SAH from 2008 to 2014.

RESULTS A total of 5415 patients (3.2\%) were hospitalized with an admission diagnosis of CA in association with SAH. Independent risk factors for CA included a higher Charlson Comorbidity Index score, hospitalization in a small or nonteaching hospital, and a Medicaid or self-pay payor status. Compared with patients with SAH and not CA, patients with CA-SAH had a higher mean NIS Subarachnoid Severity Score (SSS) \pm SD $(1.67 \pm 0.03$ vs $1.13 \pm 0.01, p<0.0001)$ and a vastly higher mortality rate ( $82.1 \%$ vs $18.4 \%, p<0.0001)$. In a multivariable model, age, NIS-SSS, and CA all remained significant independent predictors of mortality. Approximately $18 \%$ of patients with CA-SAH survived and were discharged to a rehabilitation facility or home with health services, outcomes that were most predicted by chronic disease processes and large teaching hospital status.

CONCLUSIONS In the largest study of its kind, CA at onset was found to complicate roughly $3 \%$ of spontaneous SAH cases and was associated with extremely high mortality. Despite this, survival can still be expected in approximately $18 \%$ of patients.

https://thejns.org/doi/abs/10.3171/2021.12.FOCUS21650

KEYWORDS cardiac arrest; subarachnoid hemorrhage; SAH

$\mathrm{S}$ UBARACHNOID hemorrhage (SAH) is a life-threatening condition with a hospital mortality rate of approximately $20 \%$ to $30 \% .^{1,2}$ In addition, another $10 \%$ to $15 \%$ of patients with SAH are estimated to die before arrival to the hospital. ${ }^{3,4}$ In the majority of these cases, patients experience cardiopulmonary arrest (CA) at the time of the initial hemorrhage. The frequency with which unconscious patients presenting with CA are found to have SAH ranges widely from $4 \%$ to $16 \% .^{5-7}$ Conversely, an estimated $3 \%$ to $11 \%$ of patients admitted for SAH experience CA in the prehospital phase. ${ }^{8,9}$ Cases of SAH associated with out-of-hospital CA are known to have a dismal

ABBREVIATIONS CA = cardiopulmonary arrest; $\mathrm{CCI}=$ Charlson Comorbidity Index; $\mathrm{CCP}=$ cerebral perfusion pressure; $\mathrm{DVT}=$ deep venous thrombosis; ICD-9-CM = International Classification of Diseases, 9th Revision, Clinical Modification; ICH = intracerebral hemorrhage; ICP = intracranial pressure; LOS = length of stay; NIS = National (Nationwide) Inpatient Sample; NIS-SSS = NIS Subarachnoid Severity Score; SAH = subarachnoid hemorrhage.

SUBMITTED October 28, 2021. ACCEPTED December 22, 2021.

INCLUDE WHEN CITING DOI: 10.3171/2021.12.FOCUS21650. 
prognosis, with studies finding that $0 \%$ to $9.1 \%$ of such patients survive to hospital discharge. ${ }^{8,10,11}$

$\mathrm{SAH}$ is well recognized as a cause of cardiac arrhythmia and neurogenic myocardial injury, which can manifest as sudden circulatory collapse. ${ }^{12}$ A massive surge in intracranial pressure (ICP), a resultant reduction cerebral perfusion pressure (CPP), and primary injury to the hypothalamus and brainstem vasomotor centers are thought to trigger the cascade of events leading to CA. ${ }^{13-16}$ The catecholamine surge that results from catastrophic SAH is thought to contribute as well to myocardial dysfunction in the form of neurogenic stunned myocardium, which is known to affect $10 \%$ to $30 \%$ of patients with SAH. ${ }^{17,18}$ By contrast, CA due to ventricular fibrillation, or a shockable rhythm, appears to be rare in the acute stage of SAH.,13,19 In those patients with underlying cardiac disease as a contributing factor to CA, survival seems more likely. ${ }^{7,20}$

There is a lack of large-scale population data on the impact of CA after SAH on mortality, and the discharge dispositions of those who do survive. To date, our understanding of this clinical syndrome is primarily based on smaller cohort and database studies. ${ }^{6,21,22}$ We sought to utilize a nationwide hospital database, conducting the largest investigation of its kind, to gain better understanding of clinical outcomes in patients with CA-SAH.

\section{Methods \\ Study Design}

We obtained de-identified data from the National (Nationwide) Inpatient Sample (NIS), a component of the Healthcare Cost and Utilization Project (HCUP), from 2008 to 2014. This publicly available database represents a $20 \%$ stratified sample of all discharges from US community hospitals, involving more than $97 \%$ of the US population. ${ }^{23,24}$ The IRB at our institution approved this study and waived the requirement for patient consent given that the data are de-identified.

\section{Patient Population}

We identified patients meeting inclusion criteria using previously validated International Classification of Diseases, 9th Revision, Clinical Modification (ICD-9-CM) codes. Patients were included if they were diagnosed with nontraumatic SAH (ICD-9-CM 430), CA with the cause unspecified (ICD-9-CM 427.5), and CA due to an underlying condition (ICD-9-CM 427.5). Patients were excluded if they were younger than 18 years or had missing demographic data. Patients were also excluded if they had a diagnosis of traumatic SAH (ICD-9-CM 852.1-852.9).

\section{Measurements}

The primary outcomes of this study were survival and discharge disposition in patients with SAH and CA. Secondary outcomes included risk factors for the development of CA and mortality. For patient dispositions, "other" accounts for discharges to places other than home, including discharge to a short-term rehabilitation facility, skilled nursing facility, intermediate care facility, another type of acute care facility, home healthcare, and against medical advice. We collected data on patient demographic and clinical variables; past medical history variables, including seizure disorder, alcohol use, drug use, smoking history, and hypertension; hospital complication variables, including intracerebral hemorrhage (ICH), cerebral edema, cardiogenic shock, acute respiratory distress syndrome, gastric stress ulcer, platelet and coagulation disorders, pulmonary embolism, deep venous thrombosis (DVT), meningitis or ventriculitis, pneumonia, and electrolyte disorders; and treatment variables, including decompressive hemicraniectomy and aneurysm treatment (surgical, endovascular, or none). These clinical variables were used to evaluate patient trajectories and were analyzed for their association with CA and as potential outcome predictors.

SAH severity was evaluated using the NIS Subarachnoid Severity Score (NIS-SSS). ${ }^{25}$ This validated score uses clinical data to approximate disease severity. The NIS-SSS correlates with the Hunt and Hess grading scale and has equivalent value for predicting 6-month outcomes based on the modified Rankin Scale. ${ }^{26,27}$ Variables captured by the NIS-SSS include coma (ICD-9-CM codes 780.01 and 780.03), stupor $(780.02,780.09)$, paresis/plegia (438.2-438.53, 781.4), aphasia (438.1-438.89), cranial nerve deficits (378.5-378.56, 379.4-379.43), CSF shunting (02.31-02.39), and mechanical ventilation (96.04, 96.796.72). The severity of comorbidities was assessed by the Charlson Comorbidity Index (CCI). ${ }^{23} \mathrm{CCI}$ is a scoring system that quantifies the burden of comorbid conditions, with scores ranging from 0 to 33 . In addition to patientlevel variables, we also analyzed hospital size and teaching hospital status.

\section{Statistical Analysis}

The software used for performing statistical analysis was SAS 9.4 (SAS Institute Inc.). The chi-square test for categorical variables and Student t-test were used to perform a univariate analysis on a variety of patient and hospital characteristics such as patient demographics (age, sex, race, and payor status), treatment management (clipping and coiling), teaching hospital status, hospital size, and hospital location. Because of the large number of statistical tests performed, $\mathrm{p}$ values $<0.05$ were considered significant.

\section{Results}

\section{Demographics and Clinical Course}

Using the NIS database, 170,869 patients were identified as having been hospitalized due to nontraumatic SAH from 2008 to 2014. Of those patients, 5415 (3.2\%) were hospitalized for CA in association with SAH. Patients with CA-SAH were younger $(p=0.003)$ and less often hypertensive than those with non-CA-SAH ( $p<0.0001)$, although they had more medical comorbidities, as captured by the CCI ( $\mathrm{p}<0.0001)$, and higher rates of illicit drug use and smoking ( $\mathrm{p}=0.01$ and $\mathrm{p}=0.001$, respectively) (Table 1).

\section{Hospital and Insurance Characteristics}

Patients with CA-SAH were significantly more likely 
TABLE 1. Patient demographics, hospital characteristics, and outcomes

\begin{tabular}{|c|c|c|c|c|}
\hline Variable & Total & $\mathrm{CA}$ & No CA & p Value \\
\hline No. of pts & 170,869 & 5415 & 165,454 & \\
\hline Mean age, yrs & $59.0 \pm 0.14$ & $57.6 \pm 0.45$ & $59.0 \pm 0.15$ & 0.003 \\
\hline Female sex & 62.3 & 63.8 & 62.2 & 0.25 \\
\hline Race \& ethnicity & & & & 0.14 \\
\hline White & 55.4 & 51.9 & 55.6 & \\
\hline Black & 13 & 14.4 & 13 & \\
\hline Hispanic & 10.4 & 10.6 & 10.4 & \\
\hline Other & 8.6 & 10.2 & 8.6 & \\
\hline Hypertension & 64.7 & 56.4 & 64.9 & $<0.0001$ \\
\hline Mean $\mathrm{CCl}$ score & $1.85 \pm 0.01$ & $1.94 \pm 0.05$ & $1.85 \pm 0.01$ & $<0.0001$ \\
\hline \multicolumn{5}{|l|}{ Social history } \\
\hline Alcohol use & 5.6 & 5.2 & 5.6 & 0.59 \\
\hline Drug use & 4.9 & 6.5 & 4.8 & 0.01 \\
\hline Smoking & 29.6 & 25.2 & 29.8 & 0.001 \\
\hline Payor status & & & & $<0.0001$ \\
\hline Private/other & 41.5 & 38.5 & 41.6 & \\
\hline Medicare & 35.3 & 31.9 & 35.4 & \\
\hline Medicaid & 12.8 & 15.4 & 12.7 & \\
\hline Self-pay/no charge & 10.2 & 13.9 & 10.1 & \\
\hline Hospital size & & & & 0.0004 \\
\hline Small/medium & 22.0 & 26.9 & 21.8 & \\
\hline Large & 78.0 & 73.1 & 78.2 & \\
\hline Hospital type & & & & $<0.0001$ \\
\hline Nonteaching & 21.5 & 32.4 & 21.1 & \\
\hline Teaching & 78.6 & 67.6 & 78.9 & \\
\hline Mean LOS, days & $11.7 \pm 0.15$ & $7.4 \pm 0.51$ & $11.9 \pm 0.15$ & $<0.0001$ \\
\hline Mean cost & $\$ 52,506 \pm \$ 1048$ & $\$ 41,889 \pm \$ 3857$ & $\$ 52,855 \pm \$ 1063$ & $<0.0001$ \\
\hline Disposition & & & & $<0.0001$ \\
\hline Home w/ or w/o services & 43.0 & 3.4 & 44.3 & \\
\hline Short- or long-term facility & 36.0 & 14.3 & 36.7 & \\
\hline Inpatient mortality & 20.4 & 82.1 & 18.4 & \\
\hline Other & 0.6 & 0.2 & 0.6 & \\
\hline
\end{tabular}

to be hospitalized in smaller, nonteaching hospitals than patients with SAH alone ( $p=0.0004$ and $p<0.0001$, respectively) (Table 1), and hospital region did not differ between the two groups. Patients with CA-SAH were also more likely to have Medicaid or be classified as uninsured/ self-pay than patients with SAH alone $(\mathrm{p}<0.0001)$.

\section{Multivariable Predictors of CA}

Negative predictors of $\mathrm{CA}$ in patients with SAH included hypertension (OR 0.733, $\mathrm{p}<0.0001$ ), a history of smoking or drug use (OR 0.851, $\mathrm{p}=0.0443$ ), and Medicare or private insurance status (OR 0.657, $\mathrm{p}<0.0001)$. Positive predictors of $\mathrm{CA}$ in patients with SAH included a higher CCI score (OR 1.07, $\mathrm{p}=0.0068)$ and small and/or nonteaching hospitals $(\mathrm{OR} 1.862, \mathrm{p}<0.0001)$ (Table 2).

\section{Complications, Treatment, and Hospital Course}

Patients with CA-SAH had significantly higher NISSSSs than patients with SAH alone ( $p<0.0001)$, with substantially greater rates of mechanical ventilation $(89 \%$ vs $36 \%$, p < 0.0001) and coma (21\% vs 7\%, p < 0.0001) driving the difference in scores. Of note, some components of the NIS-SSS were less frequent in patients with CA$\mathrm{SAH}$, including hydrocephalus, ventriculostomy, aphasia, and cranial nerve deficits, although these differences were small (Table 3).

With regard to hospital complications and procedures, patients with CA-SAH had significantly higher rates of electrolyte derangement ( $49 \%$ vs $40 \%, \mathrm{p}<0.0001)$, cerebral edema ( $25 \%$ vs $14 \%, \mathrm{p}<0.0001)$, ICH (10\% vs $7 \%$, $\mathrm{p}<0.0003)$, Takotsubo cardiomyopathy $(2.8 \%$ vs $0.7 \%$, 
TABLE 2. Multivariable predictors of CA in patients with SAH

\begin{tabular}{|c|c|c|c|c|}
\hline Variable & $\begin{array}{l}\text { Adjusted } \\
\text { OR }\end{array}$ & LL & UL & $\begin{array}{c}p \\
\text { Value }\end{array}$ \\
\hline Age & 0.998 & 0.993 & 1.003 & 0.3435 \\
\hline Hypertension & 0.733 & 0.628 & 0.856 & $<0.0001$ \\
\hline $\mathrm{CCl}$ score & 1.07 & 1.019 & 1.124 & 0.0068 \\
\hline $\begin{array}{l}\text { History of smoking \&/or drug } \\
\text { use vs no history of use }\end{array}$ & 0.851 & 0.728 & 0.996 & 0.0443 \\
\hline \multicolumn{5}{|l|}{ Payment method } \\
\hline $\begin{array}{l}\text { Medicare or private insur- } \\
\text { ance vs self-pay or Medicaid }\end{array}$ & 0.657 & 0.552 & 0.781 & $<0.0001$ \\
\hline Self-pay or Medicaid & Reference & & & \\
\hline $\begin{array}{l}\text { Other/missing vs self-pay or } \\
\text { Medicaid }\end{array}$ & 0.992 & 0.73 & 1.349 & 0.9615 \\
\hline \multicolumn{5}{|l|}{ Hospital type } \\
\hline $\begin{array}{l}\text { Small \&/or nonteaching } \\
\text { hospital vs medium to large } \\
\text { \&/or teaching hospital }\end{array}$ & 1.862 & 1.565 & 2.215 & $<0.0001$ \\
\hline $\begin{array}{l}\text { Medium to large \&/or teach- } \\
\text { ing hospital }\end{array}$ & Reference & & & \\
\hline $\begin{array}{l}\text { Missing vs medium to large } \\
\text { \&/or teaching hospital }\end{array}$ & 0.923 & 0.543 & 1.568 & 0.7668 \\
\hline
\end{tabular}

$\mathrm{LL}=$ lower limit; $\mathrm{UL}=$ upper limit.

Boldface type indicates statistical significance.

$\mathrm{p}<0.0001)$, and cardiogenic shock $(6.6 \%$ vs $0.6 \%$, p < $0.0001)$ than patients with SAH alone. Complications that occurred less in patients with CA-SAH included meningitis $(1.0 \%$ vs $2.3 \%, \mathrm{p}=0.002)$, ventriculitis $(0.8 \%$ vs $1.8 \%$, $\mathrm{p}=0.014)$, and DVT $(5.5 \%$ vs $7.1 \%, \mathrm{p}=0.035)($ Table 4$)$.

\section{Healthcare Burden and Outcomes}

Patients with CA-SAH had a $48 \%$ shorter hospital length of stay (LOS) compared with patients with SAH alone (mean 7.4 days vs 11.9 days, $\mathrm{p}<0.0001$ ), and sig-

TABLE 3. NIS-SSS and component model variables

\begin{tabular}{lcccc}
\hline \multicolumn{1}{c}{ Variable } & Total & CA & No CA & p Value \\
\hline Mean NIS-SSS & $1.15 \pm 0.01$ & $1.67 \pm 0.03$ & $1.13 \pm 0.01$ & $<0.0001$ \\
\hline $\begin{array}{l}\text { Mechanical } \\
\text { ventilation }\end{array}$ & 37.6 & 89.3 & 35.9 & $<0.0001$ \\
\hline Hydrocephalus & 29.2 & 23.5 & 29.4 & $<0.0001$ \\
\hline $\begin{array}{l}\text { Ventriculostomy } \\
\text { Coma }\end{array}$ & 28.3 & 25.4 & 28.4 & $\mathbf{0 . 0 3 3}$ \\
\hline Aphasia & 7.8 & 21.3 & 7.4 & $<0.0001$ \\
\hline $\begin{array}{l}\text { Cranial nerve } \\
\text { deficits }\end{array}$ & 2.3 & 3.0 & 6.5 & $<0.0001$ \\
\hline $\begin{array}{l}\text { Stupor } \\
\text { Hemiplegia or }\end{array}$ & 1.7 & 1.4 & 2.4 & 0.05 \\
paraplegia & 1.2 & 1.4 & 1.7 & 0.12 \\
\hline
\end{tabular}

Values represent the percentage of patients or mean \pm SD unless indicated otherwise. Boldface type indicates statistical significance.
TABLE 4. Complications and procedures

\begin{tabular}{lrrrc}
\hline \multicolumn{1}{c}{ Variable } & Total & CA & No CA & p Value \\
\hline Electrolyte derangement & 40.1 & 48.9 & 39.9 & $<0.0001$ \\
\hline Cerebral edema & 13.9 & 25.3 & 13.5 & $<0.0001$ \\
\hline Seizures & 10.7 & 10.9 & 10.7 & 0.84 \\
\hline PEG & 7.7 & 6.4 & 7.7 & 0.09 \\
\hline Tracheostomy & 7.0 & 7.3 & 6.9 & 0.61 \\
\hline DVT & 7.0 & 5.5 & 7.1 & $\mathbf{0 . 0 3 5}$ \\
\hline ICH & 6.9 & 9.6 & 6.8 & $\mathbf{0 . 0 0 0 3}$ \\
\hline Ischemic stroke & 6.1 & 4.7 & 6.1 & 0.07 \\
\hline Hematological dysfunction & 5.5 & 6.0 & 5.5 & 0.45 \\
\hline ARDS & 4.1 & 3.7 & 4.1 & 0.55 \\
\hline Craniotomy & 3.9 & 2.8 & 3.9 & 0.054 \\
\hline Meningitis & 2.3 & 1.0 & 2.3 & $\mathbf{0 . 0 0 2}$ \\
\hline Ventriculitis & 1.8 & 0.8 & 1.8 & $\mathbf{0 . 0 1 4}$ \\
\hline Pulmonary embolism & 1.0 & 1.5 & 0.9 & 0.06 \\
\hline Takotsubo cardiomyopathy & 0.8 & 2.8 & 0.7 & $<0.0001$ \\
\hline Cardiogenic shock & 0.8 & 6.6 & 0.6 & $<0.0001$ \\
\hline
\end{tabular}

ARDS = acute respiratory distress syndrome; PEG = percutaneous enterogastrostomy.

Values represent the percentage of patients unless indicated otherwise. Boldface type indicates statistical significance.

nificantly lower hospital costs (mean $\$ 41,889$ vs $\$ 52,855$, $\mathrm{p}<0.0001$ ) (Table 1). Patients with CA-SAH were significantly less likely to be discharged home $(3.4 \%$ vs $44.3 \%$, $\mathrm{p}<0.0001)$ or to an acute rehabilitation facility $(14.3 \% \mathrm{vs}$ $36.7 \%, \mathrm{p}<0.0001)$, and were more than four times more likely to die in the hospital ( $82.1 \%$ vs $18.4 \%$, p < 0.0001$)$. Predictors of mortality in the overall SAH population included older age, higher NIS-SSS, drug use, and cardiac arrest $(\mathrm{p}<0.0001)$ (Table 5).

A history of hypertension, smoking, and treatment at a large or teaching hospital were protective against mortality $(\mathrm{p}<0.0001)$ (Table 5). Among patients who experienced CA after SAH, the NIS-SSS was the only significant predictor of mortality $(\mathrm{p}=0.0075)$.

\section{Single-Variable Predictors of Survival}

Demographic and medical comorbidity predictors of survival in patients with CA-SAH included smoking (OR 1.187, 95\% CI 1.157-1.719; p < 0.0001) and Black race (OR 1.161, 95\% CI 1.116-1.208; $\mathrm{p}<0.0001)$. Patients were more likely to survive SAH with concomitant CA if they were admitted to a large hospital system (OR 1.096, 95\% CI 1.066-1.176; $\mathrm{p}<0.0001$ ) or teaching hospital (OR $1.138,95 \%$ CI 1.106-1.170; $p<0.0001)$. Development of ventriculitis during the hospital stay was predictive of survival as well (OR 2.009, 95\% CI 1.684-2.396; $p<0.0001)$ (Table 6).

\section{Discussion}

Overall, 3.2\% of the SAH patient population experienced $\mathrm{CA}$ at onset, an incidence rate that is at the lower end of prior reports ranging from $3 \%$ to $11 \%{ }^{8,9}$ The mor- 
TABLE 5. Predictors of mortality in the entire study population

\begin{tabular}{lcccr}
\hline \multicolumn{1}{c}{ Variable } & OR & LL & UL & p Value \\
\hline Age & 1.031 & 1.028 & 1.033 & $<0.0001$ \\
\hline F vs M & 0.945 & 0.888 & 1.005 & 0.0724 \\
\hline Race \& ethnicity & & & & \\
\hline$\quad$ White & Reference & & & \\
\hline$\quad$ Black & 0.866 & 0.786 & 0.955 & 0.0039 \\
\hline$\quad$ Hispanic & 0.899 & 0.802 & 1.007 & 0.0659 \\
\hline$\quad$ Other & 1.113 & 0.985 & 1.258 & 0.0856 \\
\hline NIS-SSS & 1.773 & 1.73 & 1.816 & $<0.0001$ \\
\hline Hypertension & 0.778 & 0.727 & 0.832 & $<0.0001$ \\
\hline Alcohol use & 0.938 & 0.817 & 1.076 & 0.3593 \\
\hline Drug use & 1.396 & 1.209 & 1.612 & $<0.0001$ \\
\hline Smoking & 0.739 & 0.688 & 0.794 & $<0.0001$ \\
\hline CA & 21.012 & 17.573 & 25.124 & $<0.0001$ \\
\hline Large (vs small/medium) & 0.863 & 0.794 & 0.939 & 0.0006 \\
hospital & & & & \\
\hline Teaching (vs nonteaching) & 0.631 & 0.580 & 0.687 & $<0.0001$ \\
hospital & & & & \\
\hline
\end{tabular}

$\mathrm{LL}=$ lower limit; $\mathrm{UL}=$ upper limit.

Boldface type indicates statistical significance.

tality rate in our patient population of those who experienced CA due to SAH was $82.1 \%$, more than four times higher than the $18.4 \%$ mortality rate of patients with SAH without CA.

The $18 \%$ survival rate among patients with SAH who experienced CA is similar to previously published reports of high mortality in this group.,10,22 On the higher end, Shapiro reported a $23 \%$ survival rate in patients with CA$\mathrm{SAH}$, with only $4 \%$ of all patients remaining in a vegetative state after treatment. ${ }^{22}$ Suzuki et al. found that of 66 patients with CA-SAH, only $9.1 \%$ survived to discharge, and the majority were in severe condition or a vegetative state. ${ }^{11}$ On the lower end, Skrifvars and Parr reported that only $0 \%$ to $2 \%$ of patients with SAH who presented with out-of-hospital CA survived to discharge. ${ }^{8}$ It is important to note that previous studies of CA-SAH analyzed much smaller sample sizes than our study. The high observed mortality in our CA-SAH population is consistent with the overall mortality rate among hospitalized patients with CA in the US. ${ }^{28}$

Although most of the patients with CA-SAH who survived to hospital discharge went to a facility, a substantial proportion did relatively well; approximately $18 \%$ were discharged from the hospital. The neurological condition of these patients was presumably good, yet it was not recorded in the NIS database. This underscores the fact that CA at the onset of severe SAH does not always imply a nohope situation. Viewed another way, of the 5415 patients with CA-SAH whom we analyzed, $3 \%$ had a surprisingly good outcome and went home from the hospital, whereas another $15 \%$ were given a fighting chance at recovery after discharge. The hospital disposition of the non-CA-SAH patient population was consistent with prior large descriptive cohort studies.
TABLE 6. Predictors of survival in the entire study population

\begin{tabular}{|c|c|c|c|c|}
\hline Variable & OR & $\mathrm{LL}$ & UL & $p$ Value \\
\hline Age & 0.949 & 0.948 & 0.949 & $<0.0001$ \\
\hline F vs $M$ & 0.889 & 0.869 & 0.911 & $<0.0001$ \\
\hline \multicolumn{5}{|l|}{ Race \& ethnicity } \\
\hline White & 0.862 & 0.832 & 0.894 & $<0.0001$ \\
\hline Black & 1.161 & 1.116 & 1.208 & $<0.0001$ \\
\hline Hispanic & 0.982 & 0.941 & 1.025 & $<0.0001$ \\
\hline Other & 0.850 & 0.821 & 0.001 & 0.410 \\
\hline NIS-SSS & 0.495 & 0.489 & 0.501 & $<0.0001$ \\
\hline Hypertension & 0.976 & 0.952 & 1.001 & $<0.0001$ \\
\hline Alcohol use & 0.862 & 0.820 & 0.906 & 0.055 \\
\hline Drug use & 0.740 & 0.699 & 0.783 & $<0.0001$ \\
\hline Smoking & 1.187 & 1.157 & 1.719 & $<0.0001$ \\
\hline CA & 0.082 & 0.073 & 0.092 & $<0.0001$ \\
\hline $\begin{array}{l}\text { Large (vs small/medium) } \\
\text { hospital }\end{array}$ & 1.096 & 1.066 & 1.176 & $<0.0001$ \\
\hline $\begin{array}{l}\text { Teaching (vs nonteaching) } \\
\text { hospital }\end{array}$ & 1.138 & 1.106 & 1.170 & $<0.0001$ \\
\hline Electrolyte derangement & 0.857 & 0.836 & 0.878 & $<0.0001$ \\
\hline Cerebral edema & 0.540 & 0.520 & 0.561 & $<0.0001$ \\
\hline Seizures & 0.773 & 0.744 & 0.802 & 0.84 \\
\hline PEG & 0.417 & 0.388 & 0.449 & $<0.0001$ \\
\hline Tracheostomy & 0.713 & 0.681 & 0.769 & $<0.0001$ \\
\hline DVT & 0.698 & 0.664 & 0.733 & $<0.0001$ \\
\hline $\mathrm{ICH}$ & 0.570 & 0.541 & 0.600 & $<0.0001$ \\
\hline Ischemic stroke & 0.511 & 0.484 & 0.540 & $<0.0001$ \\
\hline Hematological dysfunction & 0.596 & 0.566 & 0.629 & $<0.0001$ \\
\hline ARDS & 0.881 & 0.827 & 0.938 & $<0.0001$ \\
\hline Craniotomy & 0.452 & 0.421 & 0.486 & $<0.0001$ \\
\hline Meningitis & 0.543 & 0.467 & 0.630 & $<0.0001$ \\
\hline Ventriculitis & 2.009 & 1.684 & 2.396 & $<0.0001$ \\
\hline Pulmonary embolism & 0.663 & 0.579 & 0.761 & $<0.0001$ \\
\hline Takotsubo cardiomyopathy & 0.845 & 0.718 & 0.995 & 0.044 \\
\hline Cardiogenic shock & 0.447 & 0.373 & 0.536 & $<0.0001$ \\
\hline
\end{tabular}

Boldface type indicates statistical significance.

We found that larger hospital volume and teaching status were predictors of better outcome in patients with CA$\mathrm{SAH}$. This reconstitutes previous literature that has shown a benefit for critically ill patients being treated in such hospital systems. Interestingly, development of ventriculitis was shown to be the single largest predictor of good outcomes in patients with CA-SAH. It has been shown that the highest mortality rates in patients with CA-SAH occurred at the onset of their SAH bleeding. Increased LOS is correlated with increased rates of survival, for this reason, and may explain the association of survival with the development of this chronic process..$^{10,11}$

We found a variety of statistically significant patient and hospital characteristics that were associated with CA at SAH onset. In univariate analysis, patients who experienced CA-SAH were significantly younger and less often 
hypertensive. Females have been reported to have a greater risk of CA with SAH in smaller series; ${ }^{5,6,17}$ however, in this larger analysis we did not confirm this finding. In previous studies it has been noted that a lack of comorbidities was associated with $\mathrm{CA}-\mathrm{SAH},{ }^{6}$ which was not confirmed by our finding of marginally higher $\mathrm{CCI}$ scores in the CASAH population.

The NIS is an administrative database that does not record the most commonly used disease severity scores for SAH: the Hunt and Hess and the World Federation of Neurological Societies grading systems. As a result, the NISSSS, which captures various ICD-9 and ICD-10 codes for common SAH comorbidities, was developed as a disease severity measure. NIS-SSSs were significantly higher in the CA cohort, which was largely driven by more frequent coding for mechanical ventilation and coma. The fact that codes for hydrocephalus, ventriculostomy, and aphasia were applied less frequently in the CA cohort exposes a potential weakness in the NIS-SSS, since these codes might be applied less frequently in comatose patients with global cerebral edema who are quickly converted to comfort measures.

We found that patients with SAH and private insurance or Medicare were less likely to experience CA than those with Medicaid or self-pay status. This parallels studies that have reported that patients with private insurance tend to have better outcomes after CA. ${ }^{29}$ Why uninsured or underinsured patients would be at risk of experiencing $\mathrm{CA}$ at the onset of SAH remains unclear. Jacobs et al. have hypothesized that patients with private insurance may be moved through the system to medical attention more quickly. ${ }^{29}$ Similarly, our findings regarding patients with SAH presenting to small- or medium-sized hospitals might reflect difficulties with emergency medical service response times in more distant and rural locales.

Illicit drug use was higher in patients with CA-SAH in our study, a finding that may not be surprising, as drug overdose is the leading cause of nontraumatic CA in younger adults. ${ }^{30}$ Interestingly, prior smoking history was found to be protective against CA at SAH onset. The reason for the observed protective effect against $\mathrm{CA}$ at $\mathrm{SAH}$ onset is unclear.

Patients were analyzed for treatments and complications during their hospital course, many of which are predictable in this patient population. Electrolyte disorders, most commonly hypo- and hypernatremia, were substantially more common in the CA-SAH population. Our results suggest that CA may contribute to impaired hypothalamic function and osmotic regulation after SAH. Cerebral edema and ICH were also significantly more common in the CA-SAH cohort. ICH results from focal bleeding into brain parenchyma at the onset of SAH and may contribute to tissue shifts that trigger CA. Global cerebral edema, thought to be the result of transient intracranial circulatory arrest followed by reperfusion, is a common feature of poor-grade SAH that predicts poor outcome. Its presence and severity are very likely increased by coexisting CA.

An increase in cardiac-related pathology was also observed in the CA-SAH cohort. Takotsubo cardiomyopathy was coded more often in patients with CA-SAH, as was cardiogenic shock. A possible explanation for this is that the catecholamine surge and subsequent cardiac injury that occurs in the setting of intracranial crisis can trigger CA, a theory that has widely been accepted. ${ }^{1,3}$ The findings of less-frequent DVT, hydrocephalus, meningitis, and ventriculitis in patients with CA-SAH were likely due to a shorter LOS, fewer interventions, and decreased survival.

Certain limitations are inherent when using the NIS database. Only information on in-hospital mortality was provided, and, therefore, findings on out-of-hospital mortality or emergency department mortality were not included in this study, which may underestimate our mortality estimates. Similarly, whether patients experienced CA during the prehospital or acute phases, as opposed to later during their intensive care unit course, was not recorded in the database and thus was not classified in our study. We presume that the majority of patients who were coded as having CA experienced it during the acute stage of the hemorrhage, although this is not known for certain. We have no data regarding the use of therapeutic temperature modulation as neuroprotective therapy. The ICD-9-CM codes that we relied on have been previously validated; however, coding errors are always a concern with any study based on NIS data. ${ }^{23}$ Errors due to sampling bias should also be considered. Moreover, we lacked many specific clinical markers that may indicate the severity of a patient's condition on hospital admission, such as Glasgow Coma Scale and modified Fisher Scale scores as well as aneurysm size and location, that may have informed the relationship between the variables observed.

\section{Conclusions}

CA in SAH is a devastating clinical event with a substantial risk of mortality. Using data from a nationally representative database, this study demonstrated that patients who experienced CA due to SAH had a significantly higher mortality rate than patients with SAH alone. Further study is needed to develop improved treatment strategies for patients with SAH who experience CA.

\section{References}

1. Ziemba-Davis M, Bohnstedt BN, Payner TD, Leipzig TJ, Palmer E, Cohen-Gadol AA. Incidence, epidemiology, and treatment of aneurysmal subarachnoid hemorrhage in 12 midwest communities. J Stroke Cerebrovasc Dis. 2014;23(5): 1073-1082.

2. Lantigua H, Ortega-Gutierrez S, Schmidt JM, et al. Subarachnoid hemorrhage: who dies, and why? Crit Care. 2015; 19:309-318.

3. Labovitz DL, Halim AX, Brent B, Boden-Albala B, Hauser WA, Sacco RL. Subarachnoid hemorrhage incidence among Whites, Blacks and Caribbean Hispanics: the Northern Manhattan Study. Neuroepidemiology. 2006;26(3):147-150.

4. Wesali S, Persson HC, Cederin B, Sunnerhagen KS. Improved survival after non-traumatic subarachnoid haemorrhage with structured care pathways and modern intensive care. Clin Neurol Neurosurg. 2015;138:52-58.

5. Mitsuma W, Ito M, Kodama M, et al. Clinical and cardiac features of patients with subarachnoid haemorrhage presenting with out-of-hospital cardiac arrest. Resuscitation. 2011; 82(10):1294-1297.

6. Kürkciyan I, Meron G, Sterz F, et al. Spontaneous subarach- 
noid haemorrhage as a cause of out-of-hospital cardiac arrest. Resuscitation. 2001;51(1):27-32.

7. Inamasu J, Nakagawa Y, Kuramae T, Nakatsukasa M, Miyatake S. Subarachnoid hemorrhage causing cardiopulmonary arrest: resuscitation profiles and outcomes. Neurol Med Chir (Tokyo). 2011;51(9):619-623.

8. Skrifvars MB, Parr MJ. Incidence, predisposing factors, management and survival following cardiac arrest due to subarachnoid haemorrhage: a review of the literature. Scand $J$ Trauma Resusc Emerg Med. 2012;20:75.

9. Hoogmoed J, de Oliveira Manoel AL, Coert BA, et al. Why do patients with poor-grade subarachnoid hemorrhage die? World Neurosurg. 2019;131:e508-e513.

10. Toussaint LG III, Friedman JA, Wijdicks EFM, et al. Survival of cardiac arrest after aneurysmal subarachnoid hemorrhage. Neurosurgery. 2005;57(1):25-31.

11. Suzuki N, Moriwaki Y, Manaka H, Hamada K. Assessing outcome of out-of-hospital cardiac arrest due to subarachnoid hemorrhage using brain CT during or immediately after resuscitation. Signa Vitae. 2010;5(2):21-24.

12. Mayer SA, Fink ME, Homma S, et al. Cardiac injury associated with neurogenic pulmonary edema following subarachnoid hemorrhage. Neurology. 1994;44(5):815-820.

13. Morris NA, Robinson D, Schmidt JM, et al. Hunt-Hess 5 subarachnoid haemorrhage presenting with cardiac arrest is associated with larger volume bleeds. Resuscitation. 2018;123: 71-76.

14. Frontera JA. Clinical trials in cardiac arrest and subarachnoid hemorrhage: lessons from the past and ideas for the future. Stroke Res Treat. 2013;2013:263974.

15. Zachariah J, Stanich JA, Braksick SA, et al. Indicators of subarachnoid hemorrhage as a cause of sudden cardiac arrest. Clin Pract Cases Emerg Med. 2016;1(2):132-135.

16. Shin J, Kim K, Lim YS, et al. Incidence and clinical features of intracranial hemorrhage causing out-of-hospital cardiac arrest: a multicenter retrospective study. Am J Emerg Med. 2016;34(12):2326-2330.

17. Kerro A, Woods T, Chang JJ. Neurogenic stunned myocardium in subarachnoid hemorrhage. J Crit Care. 2017;38:27-34.

18. Al-Mufti F, Morris N, Lahiri S, et al. Use of Intra-aortic- balloon pump counterpulsation in patients with symptomatic vasospasm following subarachnoid hemorrhage and neurogenic stress cardiomyopathy. J Vasc Interv Neurol. 2016;9(1):28-34

19. Inamasu J, Miyatake S, Tomioka H, et al. Subarachnoid haemorrhage as a cause of out-of-hospital cardiac arrest: a prospective computed tomography study. Resuscitation. 2009;80(9):977-980.

20. Wang H, Tang L, Zhang L, Zhang ZL, Pei HH. Development a clinical prediction model of the neurological outcome for patients with coma and survived 24 hours after cardiopulmonary resuscitation. Clin Cardiol. 2020;43(9):1024-1031.

21. Choi KS, Won YD, Yi HJ, Lim TH, Lee YJ, Chun HJ. Therapeutic and prognostic implications of subarachnoid hemorrhage in patients who suffered cardiopulmonary arrest and underwent cardiopulmonary resuscitation during an emergency room stay. Clin Neurol Neurosurg. 2013;115(10): 2088-2093.

22. Shapiro S. Management of subarachnoid hemorrhage patients who presented with respiratory arrest resuscitated with bystander CPR. Stroke. 1996;27(10):1780-1782.

23. Roffman CE, Buchanan J, Allison GT. Charlson Comorbidities Index. J Physiother. 2016;62(3):171.

24. 2011 Introduction to the NIS. Healthcare Cost and Utilization Project (HCUP). July 2016. Agency for Healthcare Research and Quality, Rockville, MD. Accessed September 24, 2020. https://www.hcup-us.ahrq.gov/db/nation/nis/NIS_Introduction_2011.jsp

25. Washington CW, Derdeyn CP, Dacey RG Jr, Dhar R, Zipfel GJ. Analysis of subarachnoid hemorrhage using the Nationwide Inpatient Sample: the NIS-SAH Severity Score and Outcome Measure. J Neurosurg. 2014;121(2):482-489.

26. Rankin J. Cerebral vascular accidents in patients over the age of 60. II. Prognosis. Scott Med J. 1957;2(5):200-215.

27. Hunt WE, Hess RM. Surgical risk as related to time of intervention in the repair of intracranial aneurysms. J Neurosurg. 1968;28(1):14-20.

28. Agarwal S, Presciutti A, Roth W, et al. Determinants of longterm neurological recovery patterns relative to hospital discharge among cardiac arrest survivors. Crit Care Med.2018; 46(2):e141-e150.

29. Jacobs CS, Beers L, Park S, et al. Racial and ethnic disparities in postcardiac arrest targeted temperature management outcomes. Crit Care Med. 2020;48(1):56-63.

30. Khan S, Meyers CM, Bentley S, Manini AF. Impact of targeted temperature management on ED patients with drug overdose-related cardiac arrest. J Med Toxicol. 2019;15(1): 22-29.

\section{Disclosures}

Dr. Mayer is a consultant for Idorsia Pharmaceuticals.

\section{Author Contributions}

Conception and design: Feldstein, Dominguez, Kaur, Semaan. Acquisition of data: Feldstein, Dominguez, Kaur, Patel, Dicpinigaitis, Fuentes. Analysis and interpretation of data: Feldstein, Dominguez, Kaur, Patel, Dicpinigaitis, Semaan, Fuentes, Kamal. Drafting the article: Feldstein, Dominguez, Semaan, Fuentes, Ogulnick, Ng, Rawanduzy, Kamal. Critically revising the article: Feldstein, Dominguez, Kaur, Dicpinigaitis, Ogulnick, Kamal, Pisapia, Hanft, Amuluru, Naidu, Cooper, Prabhakaran, Mayer, Gandhi, Al-Mufti. Reviewed submitted version of manuscript: Feldstein, Dominguez, Kaur, Patel, Dicpinigaitis, Semaan, Fuentes, Ogulnick, Rawanduzy, Kamal, Pisapia, Hanft, Amuluru, Naidu, Cooper, Prabhakaran, Mayer. Approved the final version of the manuscript on behalf of all authors: Feldstein. Statistical analysis: Patel, Dicpinigaitis, Ng. Administrative/technical/material support: Gandhi, Al-Mufti. Study supervision: Mayer, Gandhi, Al-Mufti.

\section{Correspondence}

Eric Feldstein: New York Medical College, Valhalla, NY. efeldste2@student.touro.edu. 